\title{
Numeric Rating Scale
}

National Cancer Institute

\section{Source}

National Cancer Institute. Numeric Rating Scale. NCI Thesaurus. Code C121548.

A psychometric response scale to assess subjective characteristics or symptoms, which relies upon a scale of numeric result values in between two fixed end-points. 